\title{
Robust Attention Deraining Network for Synchronous Rain Streaks and Raindrops Removal
}

This paper was downloaded from TechRxiv (https://www.techrxiv.org).

LICENSE

CC BY 4.0

SUBMISSION DATE / POSTED DATE

$15-12-2021 / 18-12-2021$

\section{CITATION}

Wei, Yanyan; Zhang, Zhao; Xu, Mingliang; Hong, Richang; Fan, Jicong; Yan, Shuicheng (2021): Robust Attention Deraining Network for Synchronous Rain Streaks and Raindrops Removal. TechRxiv. Preprint. https://doi.org/10.36227/techrxiv.17205848.v1

$\mathrm{DOI}$

10.36227/techrxiv.17205848.v1 


\title{
Robust Attention Deraining Network for Synchronous Rain Streaks and Raindrops Removal
}

\author{
Yanyan Wei \\ Hefei University of Technology \\ weiyanyan@mail.hfut.edu.cn \\ Richang Hong \\ Hefei University of Technology \\ hongrc.hfutegmail.com
}

\author{
Zhao Zhang \\ Hefei University of Technology \\ cszzhang@gmail.com
}

Jicong Fan

The Chinese University of Hong Kong (Shenzhen)

fanjicong@cuhk.edu.cn

\author{
Shuicheng Yan \\ Sea AI Labs \\ Yanscasea.com
}

\begin{abstract}
Synchronous Rain streaks and Raindrops Removal $\left(S R^{3}\right)$ is a very hard and challenging task, since rain streaks and raindrops are two wildly divergent real-scenario phenomena with different optical properties and mathematical distributions. As such, most of existing deep learning-based Singe Image Deraining (SID) methods only focus on one of them or the other. To solve this issue, we propose a new, robust and hybrid SID model, termed Robust Attention Deraining Network (RadNet) with strong robustenss and generalztion ability. The robustness of RadNet has two implications: (1) it can restore different degenerations, including raindrops, rain streaks, or both; (2) it can adapt to different data strategies, including single-type, superimposed-type and blended-type. Specifically, we first design a lightweight robust attention module (RAM) with a universal attention mechanism for coarse rain removal, and then present a new deep refining module (DRM) with multi-scales blocks for precise rain removal. The whole process is unified in a network to ensure sufficient robustness and strong generalztion ability. We measure the performance of several SID methods on the $S R^{3}$ task under a variety of data strategies, and extensive experiments demonstrate that our RadNet can outperform other state-of-the-art SID methods.
\end{abstract}

\section{Introduction}

Rain removal is an important task in the low-level image restoration field, since it can affect the outdoor computer vision and multimedia computing tasks. In reality,

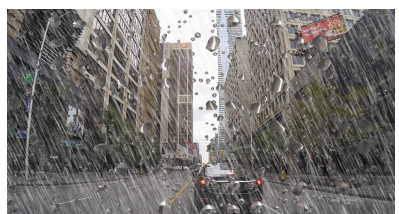

Input (17.33/0.514)

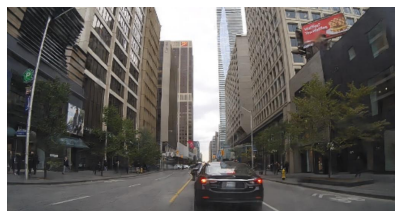

CCN $(22.73 / 0.747)$

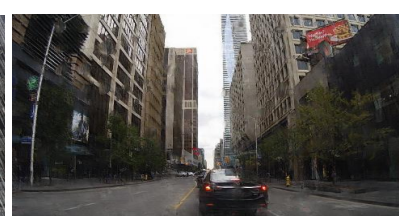

AttentGAN (22.69/0.768)

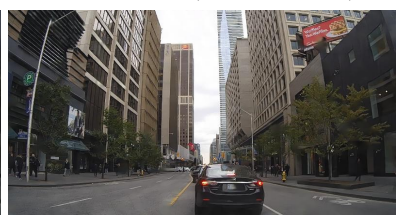

Ours $(\mathbf{3 0 . 7 2 / 0 . 9 4 3 )}$
Figure $1 . \mathrm{SR}^{3}$ task comparison (PSNR/SSIM) on an image, where (b) and (c) are the results of AttentGAN [13] and CCN [14], respectively. Our method obtains the cleanest result.

rain captured by cameras, surveillance cameras and mobile devices has two major forms, i.e., rain streak and raindrop. Due to different optical properties and mathematical distributions, rain streak removal and raindrop removal are usually treated as two different tasks. Since rain streaks appear more frequently in reality, more deep SID methods are proposed for rain streak removal. However, the rain streak removal models cannot be well generalized to the raindrop removal task and vice versa. To address the difference between the two tasks, researchers have recently proposed a new task called synchronous rain streaks and raindrops removal $\left(\mathrm{SR}^{3}\right)$ [14], which aims to remove both via a unified convolutional neural network (CNN). Next, we will describe the development of these three tasks. 


\subsection{Rain streak removal}

Rain streak images create a rain mask before the true image. For heavy rains, they may cause a haze atmosphere due to light scattering, and hence making the images blurring and haziness. Separating the rain mask from true image is an intuitional idea to solve this task. The rain streak removal problem can be modeled as follows:

$$
\mathbf{O}=\mathbf{B}+\mathbf{S}
$$

where $\mathbf{O}$ denotes a rain image that is decomposed into a rain streak component $\mathbf{S}$ and a clean background $\mathbf{B}$. This model is widely used in current SID methods [16, 19,21,24].

\subsection{Raindrop removal}

Raindrop degradation occurs when raindrop region is formed by rays of reflected light from a wider environment, which contains different imageries from those without raindrops. In most cases, the focus of camera is on the background scene, making the appearance of raindrops blur. The raindrop removal process is modeled as

$$
\mathbf{O}=\mathbf{D}+(\mathbf{1}-\mathbf{M}) \odot \mathbf{B}
$$

where $\mathbf{M}$ is a binary mask, and $\mathbf{M}(x)=1$ means the pixel $x$ is a part of rain region, and otherwise it is a part of background region. $\mathrm{D}$ is the effect brought by the raindrops, representing the complex mixture of the background information and the light reflected by the environment and passing through the raindrops adhered to a lens or windscreen. This model is also frequently used in current raindrop removal methods, such as $[13,15]$.

\subsection{Synchronous rain streak and raindrop removal}

When raindrops and rain streaks appear synchronously in the same image, they will affect each other and make the restoration task more complicated, due to different optical properties of the rain streaks and raindrops. For deep learning, it is difficult to treat the mixed data with different distributions in a unified network, which may make the network hard to converge or uneven to perform (i.e., better on either rain streaks or raindrops). $\mathrm{SR}^{3}$ task is modeled as:

$$
\mathbf{O}=\alpha \mathbf{D}+(\mathbf{1}-\mathbf{M}) \odot(\mathbf{B}+\mathbf{S})
$$

where $\alpha$ is a global atmospheric lighting coefficient. This model is used in recent $\mathrm{SR}^{3}$ method called complementary cascaded network (CCN) [14]. CCN tacked this hybrid task in a uniform network via a two-branch and two-stage strategy. However, the robustenss and generalztion ability of this divide-and-conquer strategy may be limited in real scenarios due to the independent setup of the networks and the difficulty of task un-entanglement.

\subsection{Our contributions}

Due to the difference of rain streaks and raindrops in optical properties and mathematical modeling, it is difficult to remove them at the same time. However, viewing $\mathrm{SR}^{3}$ as a simple combination of rain streak and raindrop removal is unscientific, since removing the rain streak firstly and then removing the randrop cannot recover the image completely. Since image degradation caused by rain streaks and raindrops is entangled, artificially forcing them apart will result in unpleasant results (see Figure 1). Overall, the main contributions of this paper are summarize as follows:

- To improve the robustness of $\mathrm{SR}^{3}$ task against different degenerations and data strategies, we propose a hybrid SID network, termed Robust Attention Deraining Network (RadNet), with robust attention. Firstly, we design a lightweight network with a universal attention mechanism for coarse rain removal, and then propose a deep neural network with multi-scales blocks for precise rain removal.

- We propose a new and robust attention module (RAM), which can not only restore different degenerations, including raindrops, rain streaks or both, but also can adapt to different data strategies, including single-type, superimposedtype and blended-type.

- In addition to strong robustness on benchmark data, RadNet can be potentially generalized to the real scenario, which has advantages of good rain removal, and avoiding excessive blur and artificial noise.

- Extensive experiments on both benchmarks and realworld data demonstrate the effectiveness of our method. Up to $1 \mathrm{~dB}-4 \mathrm{~dB}$ reconstruction advantage in terms of PSNR is obtained, compared with the SOTA $\mathrm{SR}^{3}$ method CCN.

\section{Related Work}

In this section, we review some representative SID methods on rain streak removal, raindrop removal, and $\mathrm{SR}^{3}$.

Rain Steak Removal Methods. Most of existing deep SID methods handles the task of rain streak removal. For example, JORDER [24] develops a multi-task deep learning architecture that learns the binary rain streak map, the appearance of rain streaks, and the clean background. PReNet [16] provides a better and simpler baseline deraining network by considering network architecture, input, output, and loss functions. Recently, RCDNet [18] proposes a model and utilize the proximal gradient descent technique to design an iterative algorithm only containing simple operators for solving the model.

Raindrop Removal Methods. To restore an image from a corrupt input, the model of [4] predicts a clean output by a specialized form of a CNN, with 3 layers and 512 neurons in each layer. AttentGAN [13] conducts a new raindrop dataset and proposes a GAN-based model to remove them by the injection of the attention map into both generative 

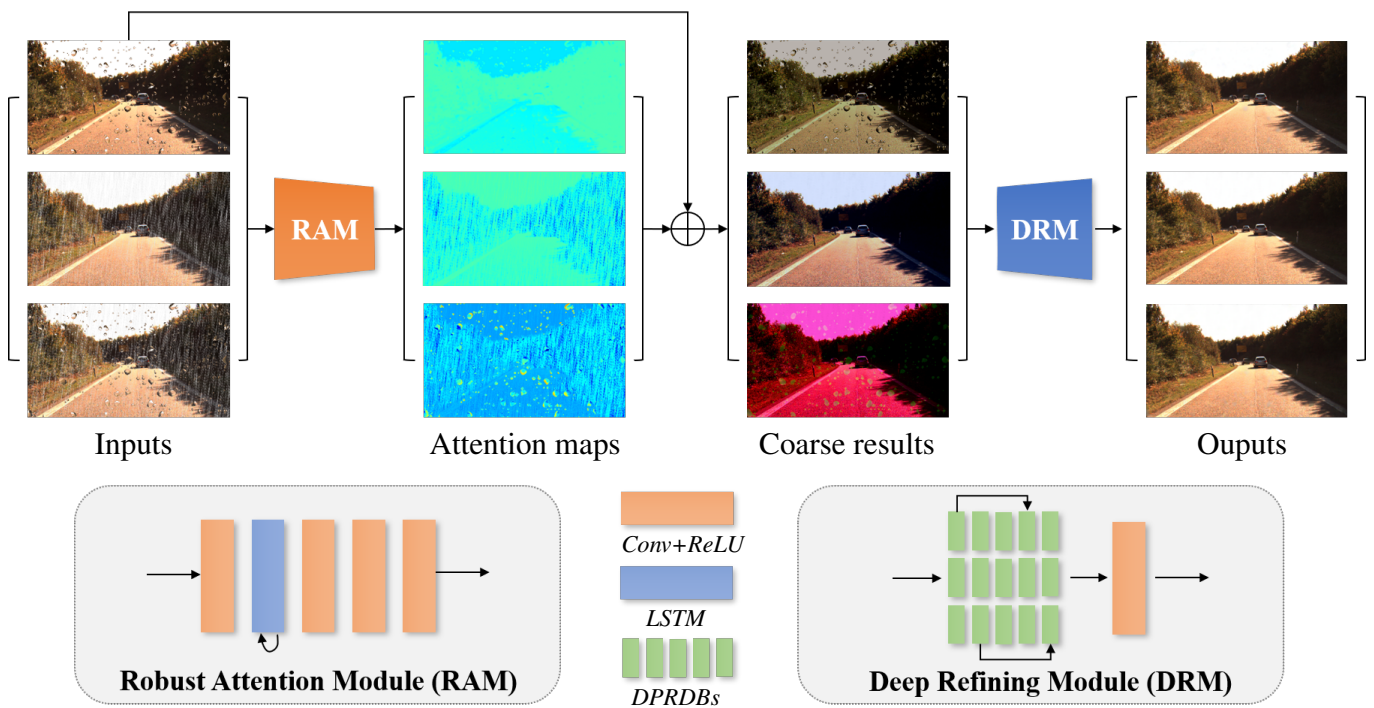

Coarse results

Ouputs

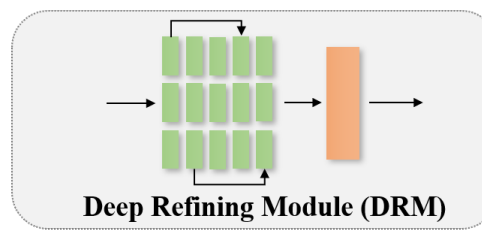

Figure 2. The proposed framework has two parts: (1) Robust Attention Module (RAM) that pays attention to various types of rain degradation; (2) Deep Refining Module (DRM) use the coarse results obtained by RAM to obtain the final refined results.

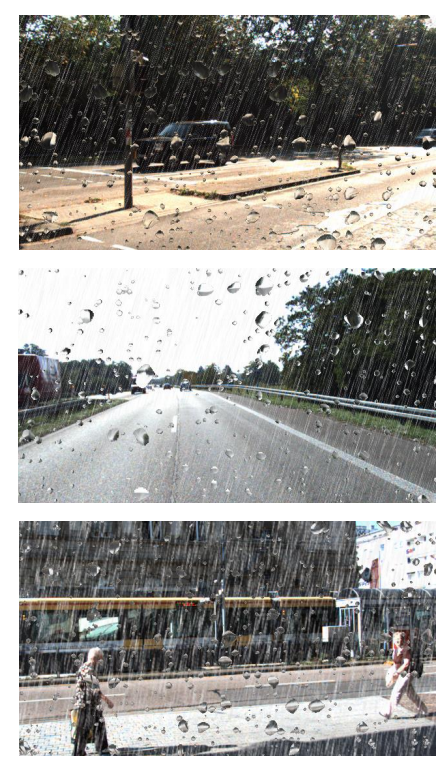

(a)
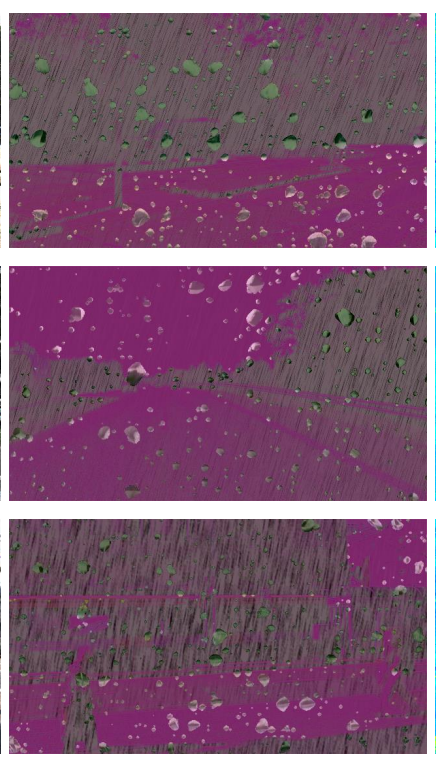

(b)
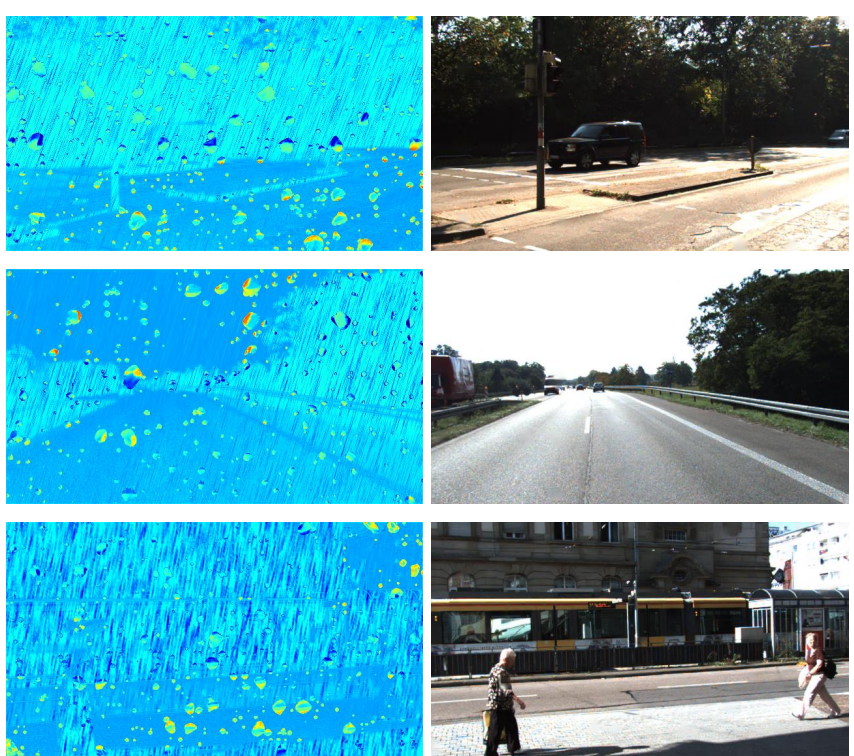

(c)

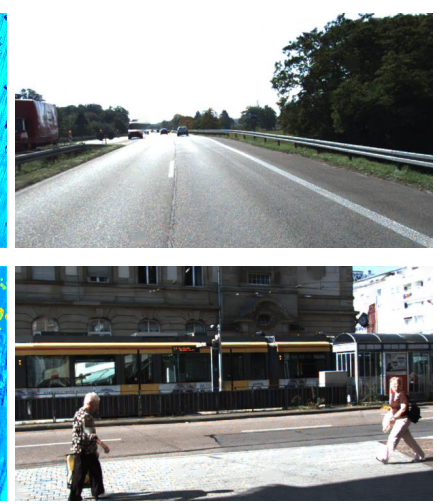

(d)

Figure 3. Illustration of the intermediate outputs of RadNet. From (a) to (d) are input, attention map by RAM, heat map of attention, and refined result by DRM, respectively.

and discriminative networks. A double attention mechanism is also introduced [15], which concurrently guides the $\mathrm{CNN}$ by shape-driven attention and channel re-calibration.

Synchronous Removal Methods. Rain streak and raindrop are two different phenomena and have a large difference between their distributions. Hence, removing both of them through a unified CNN is challenging. Recently, CCN [14] is proposed to remove the rain streaks and raindrops in a complementary fashion. $\mathrm{CCN}$ uses the NAS to adaptively find an optimal architectures. They also construct a new rain dataset RainDS which includes the rain images in dif- ferent types and their corresponding rain-free ground-truth, including rain streak only, raindrop only, and both. However, using two-stage and two-branch is not a robust way in synchronous deraining. Besides, NAS can only optimize the results to a certain extent, and the overall rain removal performance is determined by the structure and property of the network itself. Therefore, to solve this problem, we propose a robust model to remove the rain streaks and raindrops synchronously in a truly unified CNN framework for better generalization abiltiy. 
Table 1. Evaluation results in terms of PSNR and SSIM metrics under single-type data strategy.

\begin{tabular}{|c|c|c|c|c|c|c|c|}
\hline \multirow{2}{*}{ Method } & \multicolumn{4}{|c|}{ Rain streak } & \multicolumn{3}{|c|}{ Raindrop } \\
\hline & Rain200H & Rain200L & RS_syn & RS_real & RainDrop & RD_syn & RD_real \\
\hline AttentGAN [13] & - & - & - & - & $30.55 / 0.902$ & $27.25 / 0.910$ & $22.02 / 0.709$ \\
\hline DetailNet [5] & $26.17 / 0.805$ & $34.43 / 0.959$ & $31.03 / 0.916$ & $26.08 / 0.860$ & - & - & - \\
\hline RESCAN [12] & $26.60 / 0.904$ & $37.07 / 0.983$ & $38.92 / 0.982$ & $26.41 / 0.878$ & - & - & - \\
\hline PReNet [16] & $28.08 / 0.889$ & $36.76 / 0.981$ & $39.12 / 0.989$ & $26.17 / 0.878$ & - & - & - \\
\hline DRD-Net [2] & $28.16 / 0.921$ & $37.15 / 0.983$ & $30.70 / 0.897$ & $23.83 / 0.720$ & - & - & - \\
\hline Pix2pix [9] & $23.96 / 0.736$ & $30.24 / 0.893$ & $28.22 / 0.820$ & $25.19 / 0.717$ & $25.50 / 0.825$ & $24.71 / 0.803$ & 20.73/0.619 \\
\hline CCN [14] & $29.12 / 0.922$ & $37.94 / 0.984$ & $35.12 / 0.970$ & $26.83 / 0.821$ & $31.34 / 0.951$ & $33.29 / 0.975$ & $24.81 / 0.758$ \\
\hline Ours & $30.08 / 0.935$ & $38.68 / 0.990$ & 39.69/0.991 & 27.52/0.887 & 31.88/0.958 & $35.49 / 0.982$ & 25.32/0.845 \\
\hline
\end{tabular}

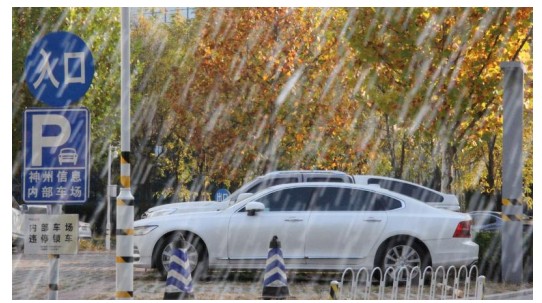

Input $(21.83 / 0.807)$

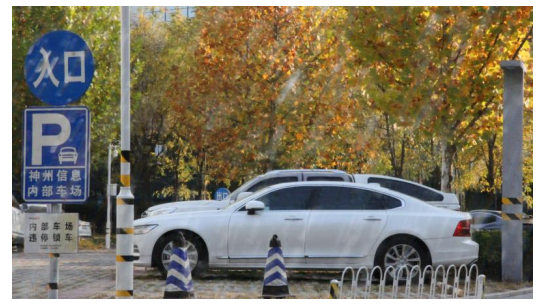

RESCAN (26.14/0.906)

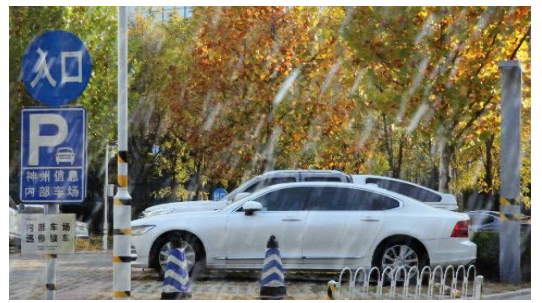

AttentGAN (23.04/0.845)

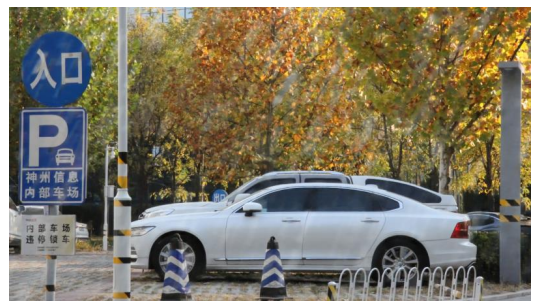

PReNet (25.63/0.914)

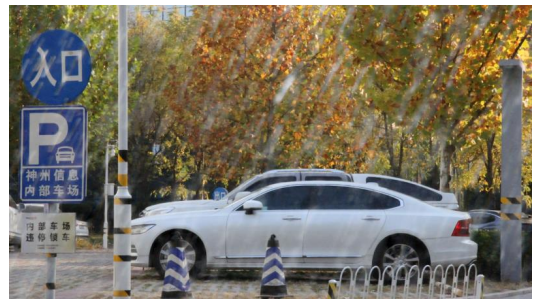

DetailNet (25.07/0.878)

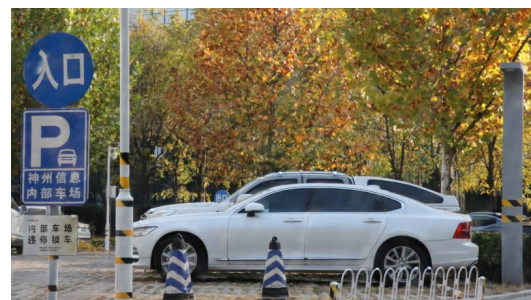

Ours $(\mathbf{2 7 . 1 1 / 0 . 9 3 2 )}$

Figure 4. Deraining comparison under single-type data strategy (i.e., RS_real) with state-of-art SID methods.

Table 2. Evaluations under superimposed-type data strategy.

\begin{tabular}{ccc}
\hline Method & RDS_syn & RDS_real \\
\hline AttentGAN [13] & $25.04 / 0.826$ & $20.94 / 0.649$ \\
\hline DetailNet [5] & $26.68 / 0.824$ & $22.33 / 0.730$ \\
RESCAN [12] & $31.66 / 0.938$ & $21.50 / 0.736$ \\
PReNet [16] & $32.87 / 0.962$ & $22.64 / 0.773$ \\
DRD-Net [2] & $22.91 / 0.699$ & $20.10 / 0.604$ \\
\hline Pix2pix [9] & $23.91 / 0.718$ & $20.33 / 0.562$ \\
CCN [14] & $32.16 / 0.950$ & $23.09 / 0.705$ \\
Ours & $\mathbf{3 4 . 1 9 / 0 . 9 7 1}$ & $\mathbf{2 4 . 3 8 / 0 . 8 5 8}$ \\
\hline
\end{tabular}

\section{Proposed SR ${ }^{3}$ Method}

In this section, we introduce the proposed RadNet framework in detail (see Figure 2). As can be seen, there are two primary parts, i.e., Robust Attention Module (RAM) and Deep Refining Module (DRM). RAM can pay attention to different rain degradation phenomena with a robust atten- tional mechanism and perform coarse rain removal results. DRM uses a multi-stream CNN to perform refined rain removal. A hybrid loss constrains the whole network.

\subsection{Robust Attention Module (RAM)}

LSTM [3] has been demonstrated to be very useful in many computer vision tasks, e.g., image deraining and dehazing. Following $[13,16,26]$, we also use the $L S T M$ unit and residual block [6] to construct our robust attention module RAM, which can pay attention to different rain degradation phenomena in different data strategies. A exmaples of the attention maps extracted by RAM is shown in Figure 3. We see that RAM can focus on most regions with rain streaks, raindrops, and both. We subtract the attention map from the original rain images to obtain coarse rain removal results, which are used as the input of DRM. RAM can reduce the abominable influence caused by the divergent distribution of different degradation phenomena and facilitate the subsequent DRM deraining.

The structure of RAM is shown in the left bottom of Fig- 


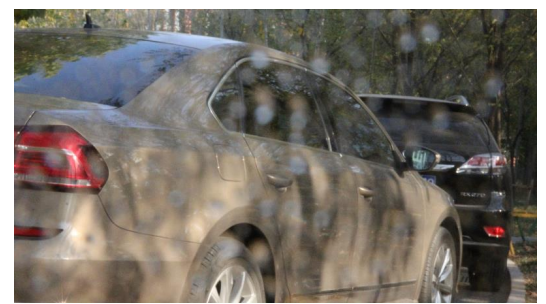

Input $(22.82 / 0.809)$

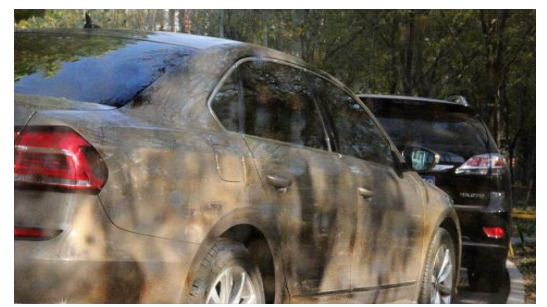

AttentGAN (21.48/0.797)

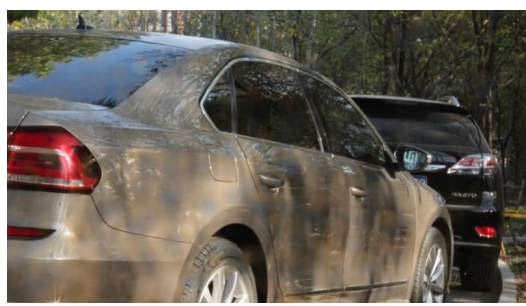

Ours $(\mathbf{2 3 . 7 6 / 0 . 8 4 6 )}$

Figure 5. Deraining comparison under single-type data strategy (i.e., RD_real) with state-of-art SID methods.

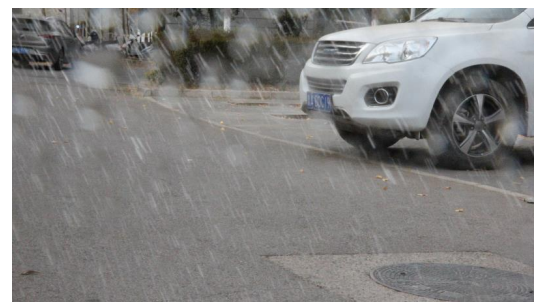

Input $(22.77 / 0.829)$

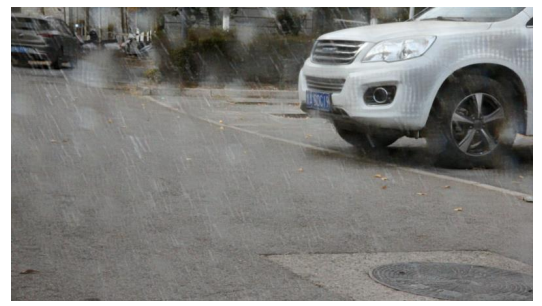

RESCAN (25.76/0.850)

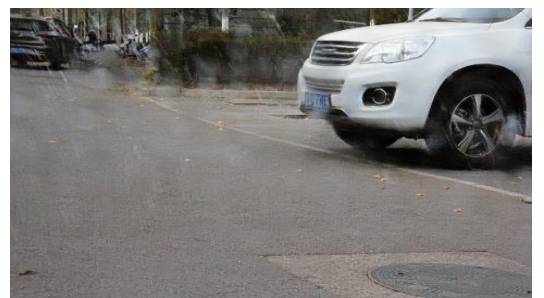

AttentGAN (26.37/0.856)

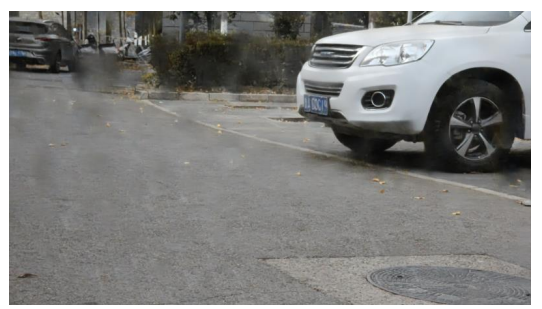

PReNet (28.09/0.898)

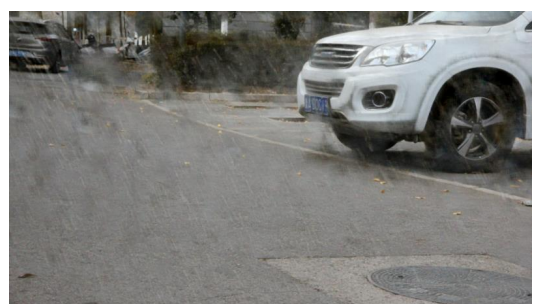

DetailNet (27.07/0.848)

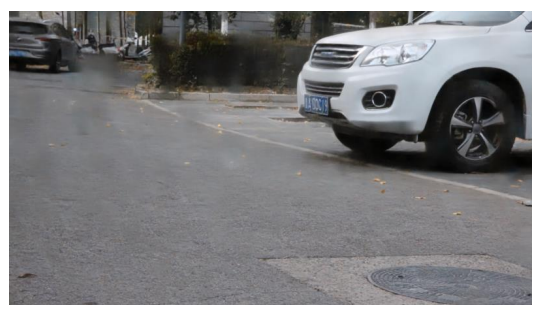

Ours $(28.65 / 0.909)$

Figure 6. Deraining comparison under superimposed-type data strategy (i.e., RDS_real) with state-of-art SID methods.

ure 2, where each stage includes a Conv $+R e L U$ operation followed by a $L S T M$ unit, and then three Conv + ReLU in the end. The LSTM unit consists of an input gate $i_{t}$, a forget gate $f_{t}$, an output gate $o_{t}$ and a cell state $c_{t}$. The interaction in each $L S T M$ unit is defined as follows:

$$
\begin{aligned}
i_{t} & =\sigma\left(W_{i}\left[X_{t}, H_{t-1}\right]+b_{i}\right), f_{t}=\sigma\left(W_{f}\left[X_{t}, H_{t-1}\right]+b_{f}\right) \\
g_{t} & =\sigma\left(W_{g}\left[X_{t}, H_{t-1}\right]+b_{g}\right), o_{t}=\sigma\left(W_{o}\left[X_{t}, H_{t-1}\right]+b_{o}\right) \\
c_{t} & =f_{t} \odot c_{t-1}+i_{t} \odot g_{t}, H_{t}=o_{t} \odot \varepsilon\left(c_{t}\right),
\end{aligned}
$$

where $X_{t}$ denotes the streak maps obtained by prepositive $t$-stage $C o n v+R e L U$ unit, $c_{t}$ denotes the cell state that will be fed to the $L S T M$ unit in the next stage, $H_{t}$ is the output of current $L S T M$ unit and will be sent to the next $C o n v+\operatorname{Re} L U$ unit, [.] is concatenate operation, $\sigma$ and $\varepsilon$ are the sigmoid and tanh functions, respectively.

\subsection{Deep Refining Module (DRM)}

In our model, RAM can be regarded as a lightweight network to obtain the coarse rain removal results. Hence, we need to use a more sophisticated network (namely, DRM) with stronger learning ability to remove the degraded remanent content after that. Traditional deep networks use simple residual blocks [6] and dense blocks [7] to extract features, but for the complex $\mathrm{SR}^{3}$ task, these blocks cannot extract appropriate features from complex data strategies. Recently, a new block called Dual Path Residual Dense Block (DPRDB) is proposed in [23], which can jointly discover new features and reuse features, and has been proved to very effective in the image restoration task with complex distributions. We therefore use DPRDB as our basic block to construct a three-branch network for in-depth deraining, since multi-scale features extracted from different kernel sizes can capture different perceptive filed information hidden in the rain images.

The structure of DRM is illustrated in Figure 2, where each branch contains five DPRDBs. The kernal sizes of each branch are set to 3,5 and 7, respectively. To avoid the information loss in deep network, we use feature shortcuts between blocks. After that, we use a Conv + ReLU operation to obtain the final refined rain removal results. A exmaples of the refined deraining result by DRM is shown in Figure 3. We see that DRM can remove most degraded region and obtain final clear output.

\subsection{Loss Function}

Due to the success of perceptual loss [10] for image restoration $[11,13,25]$, we also use it as a part of our loss 
Table 3. Evaluation results in terms of PSNR and SSIM metrics under blended-type data strategy.

\begin{tabular}{cccc}
\hline Method & RD_syn + RS_syn + RDS_syn & RD_real + RS_real + RDS_real & Rain200H + Rain200L + RainDrop \\
\hline AttentGAN [13] & $26.23 / 0.864$ & $22.51 / 0.706$ & $23.92 / 0.836$ \\
\hline DetailNet [5] & $27.06 / 0.843$ & $23.54 / 0.785$ & $23.87 / 0.823$ \\
RESCAN [12] & $33.54 / 0.956$ & $23.35 / 0.797$ & $28.63 / 0.887$ \\
PReNet [16] & $34.13 / 0.969$ & $23.96 / 0.822$ & $30.10 / 0.921$ \\
DRD-Net [2] & $32.15 / 0.884$ & $21.16 / 0.714$ & $29.32 / 0.898$ \\
\hline Pix2pix [9] & $24.77 / 0.760$ & $22.52 / 0.651$ & $24.54 / 0.760$ \\
Ours & $\mathbf{3 6 . 9 2 / 0 . 9 8 2}$ & $\mathbf{2 4 . 5 8 / 0 . 8 6 7}$ & $\mathbf{3 2 . 7 3 / 0 . 9 5 2}$ \\
\hline
\end{tabular}

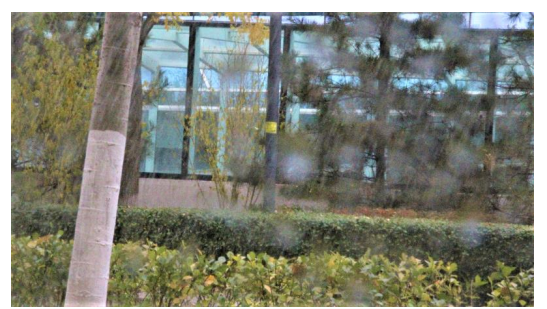

Input $(18.92 / 0.715)$

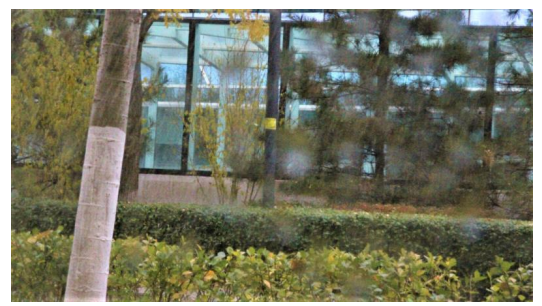

RESCAN (20.73/0.735)

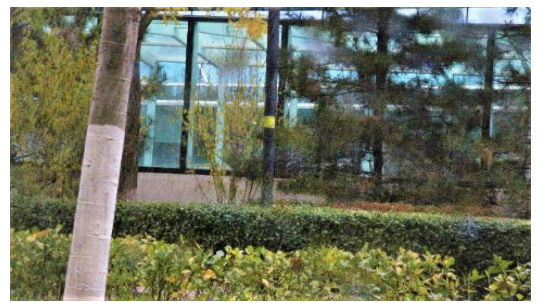

AttentGAN (21.78/0.763)

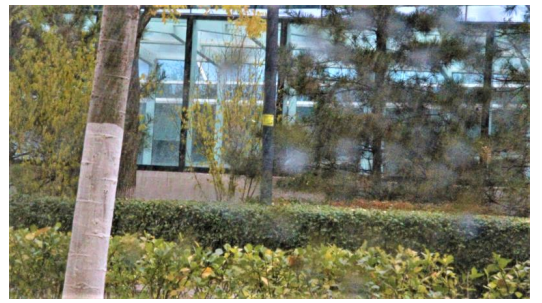

PReNet (20.10/0.760)

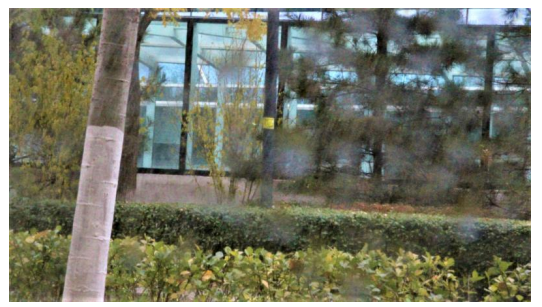

DetailNet $(20.82 / 0.730)$

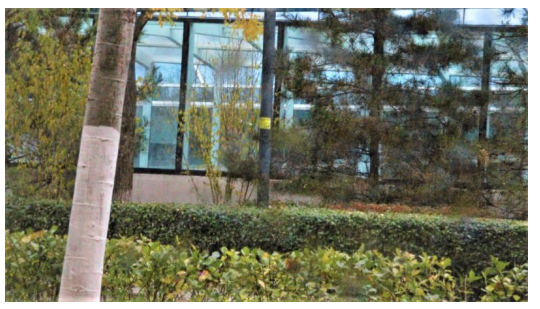

Ours (22.11/0.790)

Figure 7. Deraining comparison under blended-type data strategy (i.e., RD_real + RS_real + RDS_real) with state-of-art SID methods.

function to maintain global features. Beside, since the goal of outputting derained image is to approximates its corresponding ground truth, we directly use the SSIM loss as the other part to maintain pixel-level features. The total loss function can preserve the perpixel similarity as well as preserving the global structures:

$$
\mathcal{L}_{\text {total }}=\mathcal{L}_{p}+\lambda \mathcal{L}_{\text {ssim }}
$$

where $\mathcal{L}_{p}$ denotes the perceptual loss to minimize the difference between perceptual features, and $\mathcal{L}_{\text {ssim }}$ denotes the SSIM loss for measuring the similarity between two images. $\lambda$ is a trade-off parameter. In our method, we use the layers ReLU2_2 and ReLU2_3 of the VGG-16 model [17] as the perceptual features extracting function.

\section{Experiments}

In this section, we compare our method with other stateof-the-art SID methods under different data strategies.

\subsection{Experiment Setup}

Baselines. We divided SID methods into three categories and tested them on different data strategies:

- Raindrop removal method, i.e., AttentGAN [13].
- Rain streak removal method, i.e., DetailNet [5], RESCAN [12], PReNet [16] and DRD-Net [2].

- $\mathrm{SR}^{3}$ method, i.e., Pix2pix [9], CCN [14] and RadNet.

Robustness evaluation. We choose four datasets (i.e., Rain200H, Rain200L [24], RainDrop [13], and RainDS [14]) as the benchmark data and designed three data strategies to examine the robustness:

- Single-type data contain only one type of rain degeneration, i.e., rain streak (Rain200H, Rain200L, RS_syn, and RS_real) and raindrop (RainDrop, RD_syn, and RD_real).

- Superimposed-type data contain two type of rain degeneration in a single image, i.e., RDS_syn and RDS_real.

- Blended-type data contain two type of rain degeneration both in the same and different images, i.e., RD_syn + RS_syn + RDS_syn, RD_real + RS_real + RDS_real, and Rain200H + Rain200L + RainDrop.

Generalization evaluation. We choose two real scenario datasets (i.e., SIRR-Real [20] and Real200 [22]) as the benchmark data to examine the generalization ability:

- SIRR-Real data contain 147 real scenario images.

- Real200 data contain 200 real scenario images.

Implementation Details. We use the Pytorch platform in Python environment on a NVIDIA GeForce GTX 1080i GPU with $12 \mathrm{~GB}$ memory. Adam is used as the optimizer 


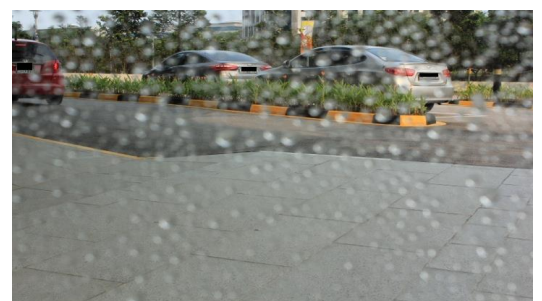

Input $(22.10 / 0.761)$

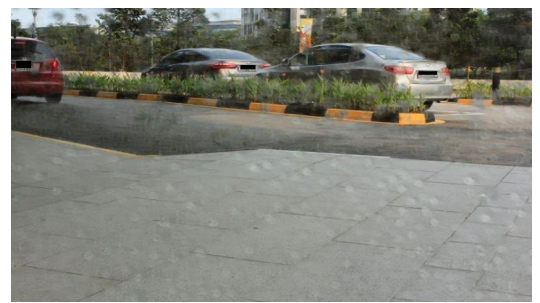

RESCAN (27.22/0.872)

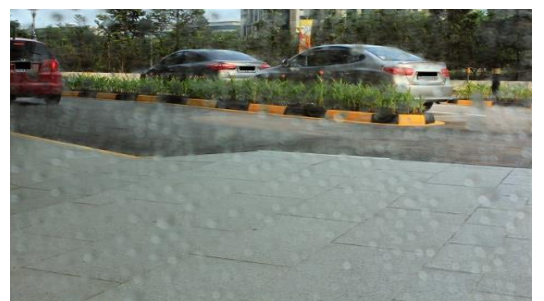

AttentGAN (26.06/0.848)

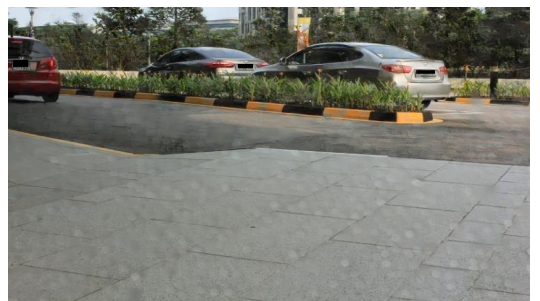

PReNet (27.44/0.903)

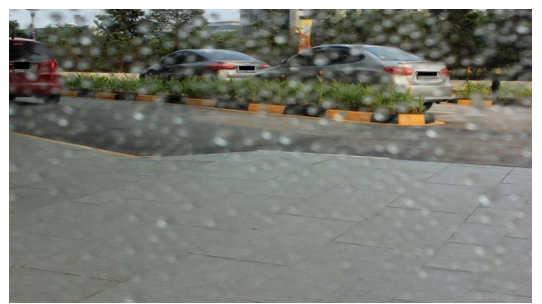

DetailNet (22.10/0.784)

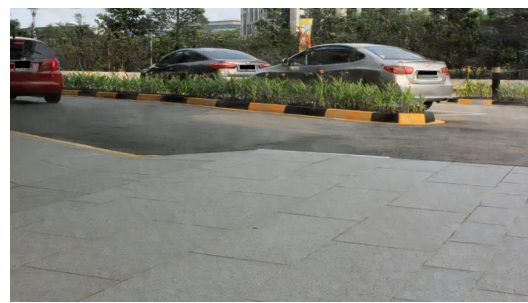

Ours $(28.34 / 0.924)$

Figure 8. Deraining comparison under blended-type data strategy (i.e., Rain200H + Rain200L + RainDrop) with state-of-art SID methods.

with an initial learning rate $1 \mathrm{e}-3$, which is decayed by multiplying 0.2 in every 30 epochs. Batch size is 16 and each image will be randomly croped to $128 \times 128$ pixels. We train 100 epochs to make the network convergence. The trade-off parameter $\lambda$ in Eqn. (5) is setting to 1. Two metrics are used for evaluations, i.e., Peak Signal to Noise Ratio (PSNR) [8] and Structural Similarity Index (SSIM) [1].

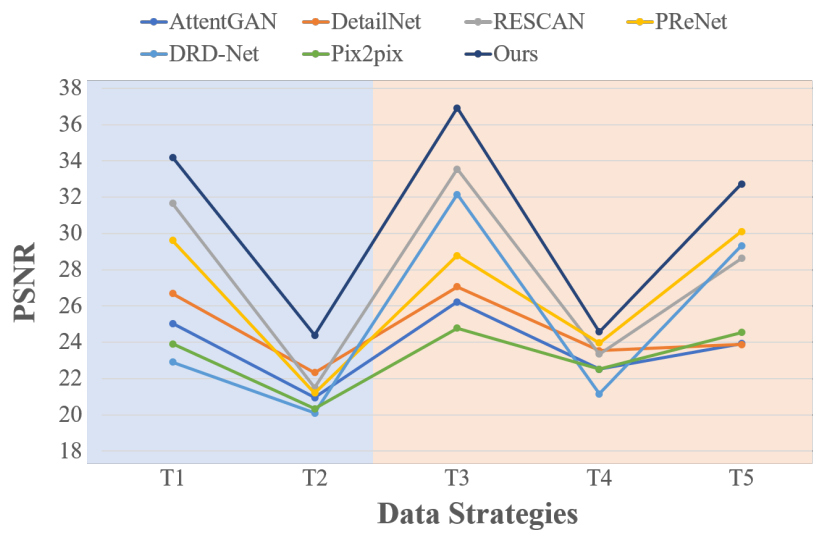

Figure 9. The performance (in terms of PSNR) of different methods under different data strategies.

\subsection{Robustness Evaluation Results}

Single-type data results. The results are described in Table 1, from which we see that our method can obtain better performance on both rain streak and raindrop data. Specifically, comparing with the rain streak removal methods (that is, DetailNet, RESCAN, PReNet and DRD-Net), our method achieves the best evaluation results, especially on RS_syn data. Furthermore, when handling the raindrop removal task, we can also obtain the best performance comparing with the related raindrop removal methods (i.e., At-
tentGAN). For a more fair comparison, we compared our method iwth two $\mathrm{SR}^{3}$ methods (i.e., Pix2pix and $\mathrm{CCN}$ ). We find that up to $4 \mathrm{~dB}$ PSNR advantages are obtained by our method over CCN in RS_syn and 2dB in RD_syn. Finally, we also illustrate some results on RS_real and RD_real in Figures 4 and 5, from which one can see our method can remove more degradation and recover clear background.

Superimposed-type data results. The restoration task under this data strategy is more difficult, which mainly focuses on processing the rain streaks and raindrops synchronously. From the numerical results in Table 2, we see that our model obtains up to $2 \mathrm{~dB}$ PSNR advantage over CCN in RDS_syn. However, all methods perform poorly on RDS_real data. This is mainly because pairs of images of the RDS dataset collected from real scenarios do not correspond at the pixel level, which brings problems to all supervised networks. But in this case, our approach still works effectively. Finally, we also illustrate some results from RDS_real in Figures 6, from which we see that our method can remove most of the rain degradation under superimposed-type data strategy.

Blended-type data results. This is the most difficult data strategy in the experiment, since the simultaneous mixing and stacking of data are disastrous for networks with weak generalization learning capabilities. However, due to the RAM and DRM, our method can handle this situation and obtain the best performance. Specifically, our model can obtain up to 2-3dB PSNR advantage over PReNet and RESCAN. Unfortunately, due to the lack of CCN code, we cannot obtain corresponding results in this part. Finally, we also illustrate some results from RD_real + RS_real + RDS_real and Rain200H + Rain200L + RainDrop in Figures 7 and 8, from which we see that most methods remains lots of rain degradation, while our method can obtain the 

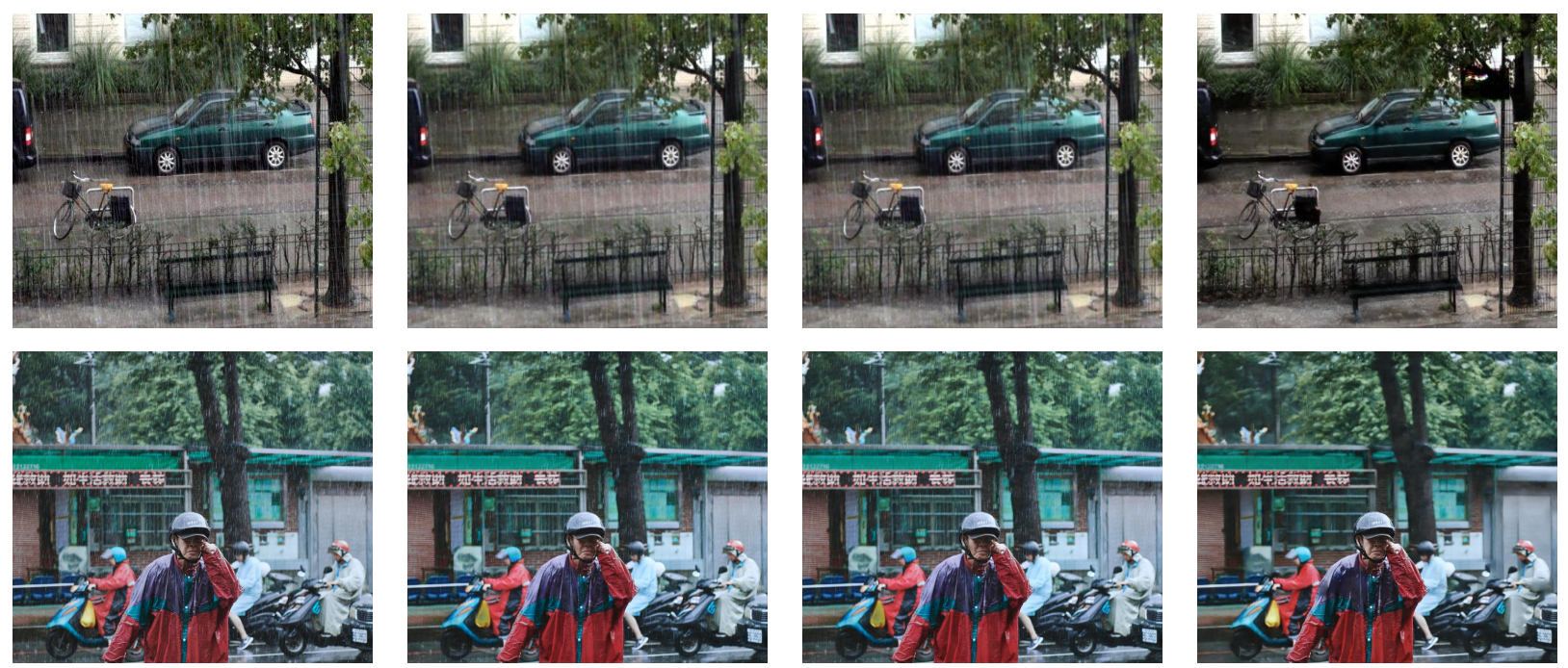

PReNet

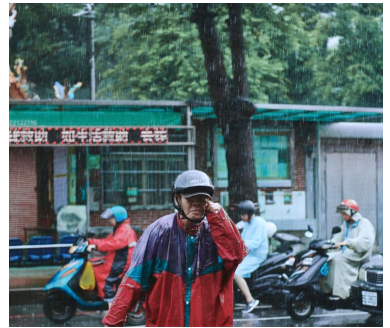

DRD-Net

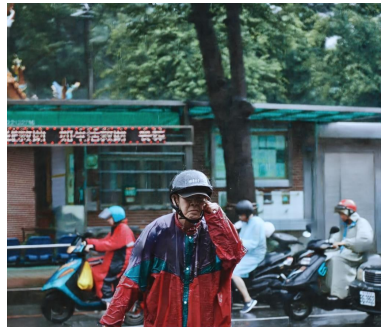

Ours

Figure 10. Deraining comparison with real scenario images (i.e., SIRR-Real and Real200) with state-of-art SID methods.

clearest image.

Remarks. To intuitively observe the performance of all methods, we use Figure 9 to show the degradation of various methods under different data strategies. Specifically, we labeled the five tests in superimposed-type and blendedtype data strategies as $\operatorname{Ti}(i=1,2,3,4,5)$. The results in the blue rectangle are the superimposed-type tests, and the results in the orange rectangle are the blended-type tests. We see from the results that our method consistently yields the best results. It is worth noting that all methods degrade on real data, mainly because the pairs of images of RDS do not correspond at the pixel level. In the future, we will study alignment technology to solve this problem.

\subsection{Generalization Evaluation Results}

Testing in real scenario images is an important way to measure the generalization of the model. Since there are no ground-truth images for comparison, we list some randomly selected results in Figure 10. It can be seen from the results that our method has the best rain removal effect, which not only remove more rain streaks, but also avoid the ambiguity caused by excessive rain removal and artificial noise.

\subsection{Ablation Study}

In this study, we mainly explore the impact of three factors, i.e., Network Module, Basic Block and Loss Function, on the deraining results of our method. Specifically, three questions are considered:

Q1: We select RAM + DRM as pipeline, whether such collocation is effective?

Q2: Why choosing DPRDB as the base block, rather than residual block and dense block?

Q3: Why adopting a mixture of the perceptual loss and SSIM loss as the loss function?
To answer the questions, we use the superimposed-type data RDS_syn in ablation study. The results are described in Tables 4, 5, and 6, respectively. Specifically,

A1: Our method with modules RAM + DRM obtains the best perfomance, and the setting with only RAM module obtains the worst performance, since the lightweight network focuses on coarse deraining and cannot learn good mapping from complex data;

A2: Our method with DPRDBs obtained the best performance. By utilizing advantages of both residual block and dense block, DPRDB can reuse previous features and exploring new features, leading to stronger learning power;

A3: The best perfomance is obtained by using both $\mathcal{L}_{p}$ and $\mathcal{L}_{\text {ssim }}$. This form of loss function can preserve the perpixel similarity as well as the global structures.

Table 4. Deraining comparison of our RadNet with different network modules.

\begin{tabular}{ccc}
\hline RAM only & DRM only & RAM + DRM \\
\hline $29.33 / 0.874$ & $32.10 / 0.928$ & $\mathbf{3 4 . 1 9 / 0 . 9 7 1}$ \\
\hline
\end{tabular}

Table 5. Comparison of our RadNet with different basic blocks.

\begin{tabular}{ccc}
\hline Residual block & Dense block & DPRDB \\
\hline $31.45 / 0.912$ & $32.27 / 0.944$ & $\mathbf{3 4 . 1 9 / 0 . 9 7 1}$ \\
\hline
\end{tabular}

Table 6. Comparison of our RadNet with different loss functions.

\begin{tabular}{ccc}
\hline $\mathcal{L}_{p}$ only & $\mathcal{L}_{\text {ssim }}$ only & $\mathcal{L}_{p}+\mathcal{L}_{\text {ssim }}$ \\
\hline $28.33 / 0.704$ & $31.89 / 0.898$ & $\mathbf{3 4 . 1 9 / 0 . 9 7 1}$ \\
\hline
\end{tabular}




\section{Conclusion}

We have explored the robustness and generalization ability in the $\mathrm{SR}^{3}$ task. Technically, we have proposed a robust and hybrid attention SID network. Compared to the recent methods that attempt to solve singe-type data, we present a new RAM+DRM pipeline that can handle various data paradigms, including single-type, superimposed-type and blended-type data. Extensive experiments and comparisons are conducted on synthetic and real rain images to evaluate the deraining performance and generalization ability of our method. The investigated results show that our model can outperform recent related methods. In future, we will further investigate how to reduce the parameters of our method, so that it can be deployed on lightweight devices.

\section{References}

[1] Alan C Brooks, Xiaonan Zhao, and Thrasyvoulos N Pappas. Structural similarity quality metrics in a coding context: exploring the space of realistic distortions. IEEE Transactions on image processing, 17(8):1261-1273, 2008. 7

[2] Sen Deng, Mingqiang Wei, Jun Wang, Yidan Feng, Luming Liang, Haoran Xie, Fu Lee Wang, and Meng Wang. Detailrecovery image deraining via context aggregation networks. In Proceedings of the IEEE/CVF Conference on Computer Vision and Pattern Recognition, pages 14560-14569, 2020. 4,6

[3] Jeffrey Donahue, Lisa Anne Hendricks, Sergio Guadarrama, Marcus Rohrbach, Subhashini Venugopalan, Kate Saenko, and Trevor Darrell. Long-term recurrent convolutional networks for visual recognition and description. In Proceedings of the IEEE conference on computer vision and pattern recognition, pages 2625-2634, 2015. 4

[4] David Eigen, Dilip Krishnan, and Rob Fergus. Restoring an image taken through a window covered with dirt or rain. In Proceedings of the IEEE international conference on computer vision, pages 633-640, 2013. 2

[5] Xueyang Fu, Jiabin Huang, Delu Zeng, Yue Huang, Xinghao Ding, and John Paisley. Removing rain from single images via a deep detail network. In Proceedings of the IEEE Conference on Computer Vision and Pattern Recognition, pages 3855-3863, 2017. 4, 6

[6] Kaiming He, Xiangyu Zhang, Shaoqing Ren, and Jian Sun. Deep residual learning for image recognition. In Proceedings of the IEEE conference on computer vision and pattern recognition, pages 770-778, 2016. 4, 5

[7] Gao Huang, Zhuang Liu, Laurens Van Der Maaten, and Kilian Q Weinberger. Densely connected convolutional networks. In Proceedings of the IEEE conference on computer vision and pattern recognition, pages 4700-4708, 2017. 5

[8] Quan Huynh-Thu and Mohammed Ghanbari. Scope of validity of psnr in image/video quality assessment. Electronics letters, 44(13):800-801, 2008. 7

[9] Phillip Isola, Jun-Yan Zhu, Tinghui Zhou, and Alexei A Efros. Image-to-image translation with conditional adversarial networks. In Proceedings of the IEEE conference on computer vision and pattern recognition, pages 1125-1134, 2017. 4, 6

[10] Justin Johnson, Alexandre Alahi, and Li Fei-Fei. Perceptual losses for real-time style transfer and super-resolution. In European conference on computer vision, pages 694-711. Springer, 2016. 5

[11] Ruoteng Li, Loong-Fah Cheong, and Robby T Tan. Heavy rain image restoration: Integrating physics model and conditional adversarial learning. In Proceedings of the IEEE Conference on Computer Vision and Pattern Recognition, pages 1633-1642, 2019. 5

[12] Xia Li, Jianlong Wu, Zhouchen Lin, Hong Liu, and Hongbin Zha. Recurrent squeeze-and-excitation context aggregation net for single image deraining. In Proceedings of the European Conference on Computer Vision (ECCV), pages 254269, 2018. 4, 6

[13] Rui Qian, Robby T Tan, Wenhan Yang, Jiajun Su, and Jiaying Liu. Attentive generative adversarial network for raindrop removal from a single image. In Proceedings of the IEEE conference on computer vision and pattern recognition, pages 2482-2491, 2018. 1, 2, 4, 5, 6

[14] Ruijie Quan, Xin Yu, Yuanzhi Liang, and Yi Yang. Removing raindrops and rain streaks in one go. In Proceedings of the IEEE/CVF Conference on Computer Vision and Pattern Recognition, pages 1-10, 2021. 1, 2, 3, 4, 6

[15] Yuhui Quan, Shijie Deng, Yixin Chen, and Hui Ji. Deep learning for seeing through window with raindrops. In Proceedings of the IEEE/CVF International Conference on Computer Vision, pages 2463-2471, 2019. 2, 3

[16] Dongwei Ren, Wangmeng Zuo, Qinghua Hu, Pengfei Zhu, and Deyu Meng. Progressive image deraining networks: a better and simpler baseline. In Proceedings of the IEEE Conference on Computer Vision and Pattern Recognition, pages 3937-3946, 2019. 2, 4, 6

[17] Karen Simonyan and Andrew Zisserman. Very deep convolutional networks for large-scale image recognition. arXiv preprint arXiv:1409.1556, 2014. 6

[18] Hong Wang, Qi Xie, Qian Zhao, and Deyu Meng. A modeldriven deep neural network for single image rain removal. In Proceedings of the IEEE/CVF Conference on Computer Vision and Pattern Recognition, pages 3103-3112, 2020. 2

[19] Tianyu Wang, Xin Yang, Ke Xu, Shaozhe Chen, Qiang Zhang, and Rynson WH Lau. Spatial attentive single-image deraining with a high quality real rain dataset. In Proceedings of the IEEE Conference on Computer Vision and Pattern Recognition, pages 12270-12279, 2019. 2

[20] Wei Wei, Deyu Meng, Qian Zhao, Zongben Xu, and Ying $\mathrm{Wu}$. Semi-supervised transfer learning for image rain removal. In Proceedings of the IEEE Conference on Computer Vision and Pattern Recognition, pages 3877-3886, 2019. 6

[21] Yanyan Wei, Zhao Zhang, Yang Wang, Mingliang Xu, Yi Yang, Shuicheng Yan, and Meng Wang. Deraincyclegan: Rain attentive cyclegan for single image deraining and rainmaking. IEEE Transactions on Image Processing, 2021. 2

[22] Yanyan Wei, Zhao Zhang, Yang Wang, Haijun Zhang, Mingbo Zhao, Mingliang Xu, and Meng Wang. Semideraingan: A new semi-supervised single image deraining 
network. In Proceedings of the IEEE International Conference on Multimedia and Expo, pages 1-6, 2021. 6

[23] Yanyan Wei, Zhao Zhang, Haijun Zhang, Richang Hong, and Meng Wang. A coarse-to-fine multi-stream hybrid deraining network for single image deraining. In 2019 IEEE International Conference on Data Mining (ICDM), pages 628-637. IEEE, 2019. 5

[24] Wenhan Yang, Robby T Tan, Jiashi Feng, Jiaying Liu, Zongming Guo, and Shuicheng Yan. Deep joint rain detection and removal from a single image. In Proceedings of the IEEE Conference on Computer Vision and Pattern Recognition, pages 1357-1366, 2017. 2, 6

[25] He Zhang, Vishwanath Sindagi, and Vishal M Patel. Image de-raining using a conditional generative adversarial network. IEEE transactions on circuits and systems for video technology, 2019. 5

[26] Hongyuan Zhu, Xi Peng, Joey Tianyi Zhou, Songfan Yang, Vijay Chanderasekh, Liyuan Li, and Joo-Hwee Lim. Singe image rain removal with unpaired information: A differentiable programming perspective. In Proceedings of the AAAI Conference on Artificial Intelligence, volume 33, pages 9332-9339, 2019. 4 